\title{
Assessment of skin dose for breast chest wall radiotherapy as a function of bolus material
}

\author{
Shu-Hui Hsu, Peter L Roberson, Yu Chen, Robin B Marsh, Lori J Pierce \\ and Jean M Moran \\ Department of Radiation Oncology, University of Michigan, Ann Arbor, MI 48109, USA. \\ E-mail: shuihsu@umich.edu,roberp1@med.umich.edu, ychen7@gmail.com, \\ marshr@med.umich.edu, ljpierce@med.umich.edu and jmmoran@med.umich.edu
}

Received 22 January 2008, in final form 20 March 2008

Published 25 April 2008

Online at stacks.iop.org/PMB/53/2593

\begin{abstract}
Skin dose assessment for chest wall radiotherapy is important to ensure sufficient dose to the surface target volume without excessive skin reaction. This study quantified changes to the surface doses as a function of bolus material for conventional and intensity modulated radiation therapy (IMRT) tangential fields. Three types of bolus materials ( $2 \mathrm{~mm}$ solid, $2 \mathrm{~mm}$ fine mesh and $3.2 \mathrm{~mm}$ large mesh Aquaplast) were compared with Superflab. Surface dose measurements were performed using an Attix parallel plate chamber in a flat solid water phantom at $0^{\circ}, 45^{\circ}$ and $70^{\circ}$ incident angles. Over-response correction factors were applied to the Attix chamber results for different incident angles. Surface dose measurements on an anthropomorphic phantom were done using a thermoluminescent dosimeter extrapolation method. Dose characteristics of Superflab and solid Aquaplast were within 2\% of solid water material. No significant differences (within 3\%) in the surface dose were found between conventional and IMRT tangential techniques. The bolus effect was large for chest wall tangential radiotherapy, with up to an $82 \%$ increase using $2 \mathrm{~mm}$ fine mesh Aquaplast. The dosimetric effect of different Aquaplast materials has been quantified in this work. These materials can be used to create a custom bolus with potentially better reproducibility of placement.
\end{abstract}

(Some figures in this article are in colour only in the electronic version)

\section{Introduction}

Accurate assessments of surface and superficial doses in radiotherapy can provide valuable information for clinical consideration to avoid near-surface recurrence while at the same time limiting severe skin toxicity, especially for breast and head-and-neck treatments. Dose at the surface is primarily due to electron contamination from the flattening filter, beam modifiers 
and air. The magnitude of the surface dose depends on the field size, angle of beam incidence, air gap and the use of beam modifiers (Biggs and Ling 1979, Gerbi et al 1987, Lamb and Blake 1998, Lopez Medina et al 2005, Petti et al 1983a, 1983b, Yang et al 2004, Zhu and Palta 1998).

Recent studies have focused on the skin dose for intensity modulated radiation therapy (IMRT) beams. For example, Lee et al (2002) identified severe skin reactions in some patients receiving IMRT for head-and-neck cancer and investigated the causes. They found that the average increase of the skin dose was about $18 \%$ due to the bolus effect of a thermoplastic mask material, and the skin doses with masks were $84 \%$ and $100 \%$ of the prescribed dose for opposed lateral and extended-field IMRT (EF-IMRT), respectively, with the skin as part of the target volume. They concluded that potential contributions to the increased skin dose are the bolus effect of the head-and-neck and shoulder mask, the use of multiple oblique beams and the inclusion of the skin as part of the target volume. Higgins et al (2007) investigated delivery technique differences and found that the maximum surface doses of head-and-neck treatment were $69 \%, 71 \%$ and $82 \%$ for bilateral fields, tomotherapy and IMRT, respectively. With respect to the impact of IMRT on surface doses, some investigators have found that IMRT itself is not contributing to greater skin dose. For example, Dogan and Glasgow (2003) found that for the $6 \times 6 \mathrm{~cm}^{2}$ field and $6 \mathrm{MV}$ x-rays, the surface doses of the IMRT field were $8 \%$ and $6 \%$ lower than those of the open field for $0^{\circ}$ and $75^{\circ}$ incidences. Yokoyama et al (2004) found that the near-surface dose ( $2 \mathrm{~mm}$ depth) of the IMRT field was $\sim 10 \%$ lower than that of the open field. Thomas and Hoole (2004) demonstrated that inverse planning, based on minimizing objective functions, can result in solutions that give high fluence to tangential beam segments near the skin surface, to counter the buildup region. They also evaluated the effects of different solutions for this problem, e.g. the use of the skin as an organ at risk, modification of the planning target volume (PTV) to avoid the skin and the use of phantom bolus.

Based on previous studies, many factors affect the doses of the skin and near-surface tumor, e.g. the use of bolus, oblique incident angle and delivery technique. The inaccuracy of calculations in the buildup region will also affect the doses given to the normal skin tissues and near-surface tumors. Therefore, it is very important to measure the dose for near-surface tumors and also to measure the dose delivered to the skin. While factors affecting the skin dose for head-and-neck treatments have been well studied, less is known about the skin dose for chest wall radiotherapy. For chest wall tangential radiotherapy, bolus is often used during the treatment course to ensure adequate dose to the target volume which includes the skin but may be removed if deemed clinically necessary when skin toxicity occurs. Accurate skin dose assessment is more challenging in this treatment geometry due to the tangential beams and the large curvature of the chest, the use of bolus materials and the combination of the entrance and exit dose. Therefore, the purpose of this study was to measure the surface dose without and with various bolus types and thicknesses for perpendicular and oblique incidences. Because the surface dose also depends on the delivery technique, doses were measured and compared for conventional and IMRT tangential techniques. The goal of these measurements was to provide clinicians information about the impact of the delivery technique on the surface dose and detailed information to aid in selection of an appropriate bolus type and thickness for the duration of therapy.

\section{Materials and methods}

In this work, the surface and near-surface doses were measured for Superflab (Med-Tec, Orange, IA) and Aquaplast (KGF Enterprise Inc., Chesterfield, MI) bolus materials in a 


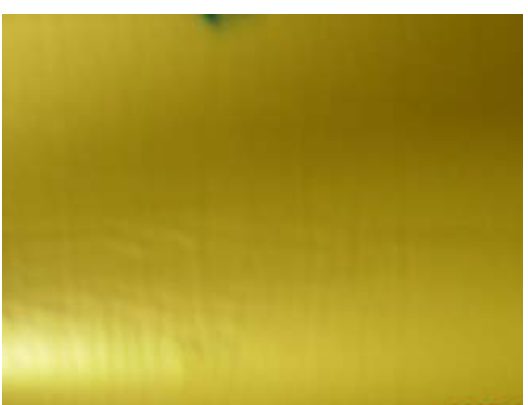

(a)

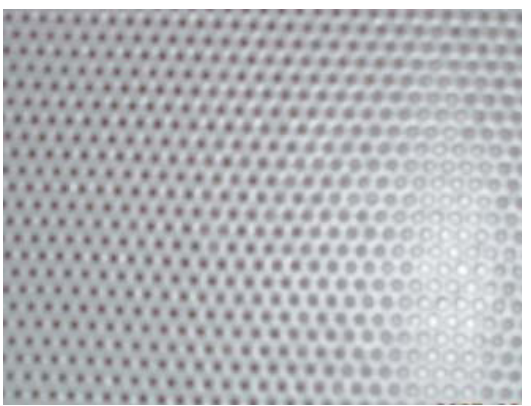

(c)

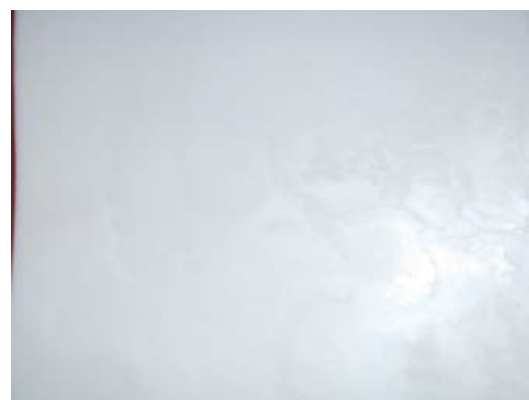

(b)

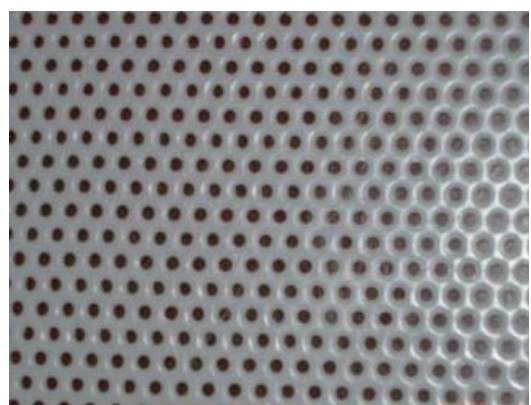

(d)

Figure 1. Bolus materials used in this study: (a) Superflab, (b) solid Aquaplast, (c) fine mesh Aquaplast and (d) large mesh Aquaplast.

Table 1. The type, thickness and perforation diameter for bolus materials used in this study. The density of Superflab and the measured density of solid Aquaplast are $1.02 \mathrm{~g} \mathrm{~cm}^{-3}$ and $1.08 \pm$ $0.02 \mathrm{~g} \mathrm{~cm}^{-3}$, respectively. The thicknesses and perforation diameters were measured using the caliper, excluding superflab materials.

\begin{tabular}{lll}
\hline Bolus materials & Thickness $(\mathrm{mm})$ & Perforation diameter $(\mathrm{mm})$ \\
\hline Superflab & 5 & None \\
Superflab & 10 & None \\
Solid Aquaplast & $1.82 \pm 0.10$ & None \\
Solid Aquaplast & $4.85 \pm 0.06$ & None \\
Fine mesh Aquaplast & $1.83 \pm 0.09$ & $0.88 \pm 0.07$ \\
Large mesh Aquaplast & $3.17 \pm 0.05$ & $2.77 \pm 0.17$ \\
Stretched solid Aquaplast & $2.06 \pm 0.13$ & None \\
Stretched fine mesh Aquaplast & $1.89 \pm 0.28$ & $1.14 \pm 0.24$ \\
Stretched large mesh Aquaplast & $3.23 \pm 0.06$ & $2.53 \pm 0.26$ \\
\hline
\end{tabular}

geometric and an anthropomorphic phantom. The thicknesses and perforation sizes (where applicable) of the bolus are shown in figure 1 and table 1. Unstretched bolus materials were used for measurements on a geometric phantom, and stretched ones were used for measurements on an anthropomorphic phantom. The slab phantom geometries were composed of solid water slabs (Gammex RMI Model 457, Middleton, WI) with total dimensions of $30 \times$ $30 \times 20$ and $40 \times 40 \times 20 \mathrm{~cm}^{3}$. An Alderson Rando anthropomorphic phantom (Radiology Support Devices Inc., Long Beach, CA) was used to simulate a chest wall treatment with obliquely incident beams. All measurements were made on a $600 \mathrm{C}$ (Varian, Palo Alto, CA), 6 MV linear accelerator equipped with a Millennium 120-leaf MLC. 
An Attix parallel plate chamber (Gammex RMI Model 449, Middleton, WI) was used to measure the dose in the slab phantom and to provide reference measurements for thermoluminescent dosimeters (TLDs). The dimensions of the Attix chamber are $1 \mathrm{~mm}$ separation, $12.7 \mathrm{~mm}$ diameter collector, $4.8 \mathrm{mg} \mathrm{cm}^{-2}$ Kapton entrance window and $40 \mathrm{~mm}$ wall diameter. The Attix chamber has low over-response and low polarity effect in the buildup region (Gerbi 1993), and can measure the dose near the surface ( $\sim 4 \mu \mathrm{m}$ window). The charge was measured with a Therapy Dosimeter Model 35040 (Chesterland, OH) or a PRM Precision Dosimeter Model SH-1 (Nashville, TN). Each measurement point represents an average of six readings for two bias voltages $(+300 \mathrm{~V}$ and $-300 \mathrm{~V})$. A custom slab piece $\left(40 \times 40 \times 2.5 \mathrm{~cm}^{3}\right)$ (Gammex RMI Solid Water) was used to hold the Attix chamber. The Attix chamber overresponds with an increasing angle of beam incidence (Gerbi 1993, Gerbi and Khan 1997). Therefore, the modified Rawlinson equation (Rawlinson et al 1992) with data from Gerbi (1993) and Gerbi and Khan (1990, 1997) was used to determine the over-response correction factors for different incident angles for the chamber. For $6 \mathrm{MV}$ x-rays, the correction factors were determined to be up to $0.7 \%, 2.3 \%$ and $4.2 \%$ (relative to the maximum dose, $D_{\max }$ ) for $0^{\circ}, 45^{\circ}$ and $70^{\circ}$ incident angles, respectively. The polarity effect $(+300 \mathrm{~V}$ and $-300 \mathrm{~V})$ was confirmed to be within $2 \%$ in the buildup region, except at the surface (up to $7 \%$ for the small field and small incident angle).

Lithium fluoride TLD chips (TLD-100, Harshaw, Solon, OH) were used with a cross section of $3.175 \times 3.175 \mathrm{~mm}^{2}$ and three thicknesses of $35 \mathrm{mil}(0.89 \mathrm{~mm}), 15 \mathrm{mil}(0.38 \mathrm{~mm})$ and $6 \mathrm{mil}(0.15 \mathrm{~mm})$. TLDs were placed in a custom holder $\left(30 \times 30 \times 1 \mathrm{~cm}^{3}\right)($ Gammex RMI Solid Water) with cavities that were machined to fit the corresponding chip thicknesses. Because of their size, TLDs are suitable dosimeters for surface dose measurements on the anthropomorphic phantom and for IMRT fields. However, a systematic error will occur if only one thickness of the TLD chip is used in the high dose gradient region. Therefore, a TLD extrapolation method was used to more accurately measure the buildup dose in this study (Kron et al 1993, 1996, Rapley 2006). Kron et al (1993) showed that the TLD response does not linearly depend on the depth of the TLD geometric center, and a linear fit could lead to overestimation at the surface. Rapley (2006) found that a second degree polynomial extrapolation provided a more accurate analysis for surface dose measurements using TLD powder extrapolation. Therefore, when doing the extrapolation for the surface and nearsurface dose, a linear fit was used with bolus but a quadratic fit was used without bolus to improve the approximation of the dose at the surface in this study (figure 2). Improved accuracy using the quadratic fit at the surface was confirmed by a comparison to the Attix chamber measurements. For all TLD extrapolation measurements in our study, three TLD chips were used for 35 mil and two TLD chips were used for both 15 and 6 mil. TLDs were consistently annealed at $400{ }^{\circ} \mathrm{C}$ for $1 \mathrm{~h}$ and $100{ }^{\circ} \mathrm{C}$ for $2 \mathrm{~h}$ prior to irradiation and $100{ }^{\circ} \mathrm{C}$ for $10 \mathrm{~min}$ after irradiation for pre-readout annealing. TLDs were read with a photomultiplier tube (PMT) bias of $\sim 800 \mathrm{~V}$ without nitrogen gas (Harshaw Model 3500, Solon, OH). Three repeated measurements were done to determine individual sensitivity factors for each TLD, and the average standard deviation of the individual sensitivity factor was $1 \%$ for the repeated measurements. TLD linearity (0-180 cGy) was assessed for each thickness of TLD, with $R$-squared of 0.999. Finally, TLDs were read in the same order to reduce the effect of PMT response decreasing with sequential readout (Yu and Luxton 1999).

The estimated errors of Attix chamber measurements were $\pm 2 \%$, including the reading variation, the error of geometric setup and the over-response correction factors. The estimated errors of TLD extrapolation methods were $\pm 3 \%$, including the error of each TLD reading corrected with its own individual sensitivity factor, the error in the dose conversion factor, the error of geometric setup and the extrapolation error. 


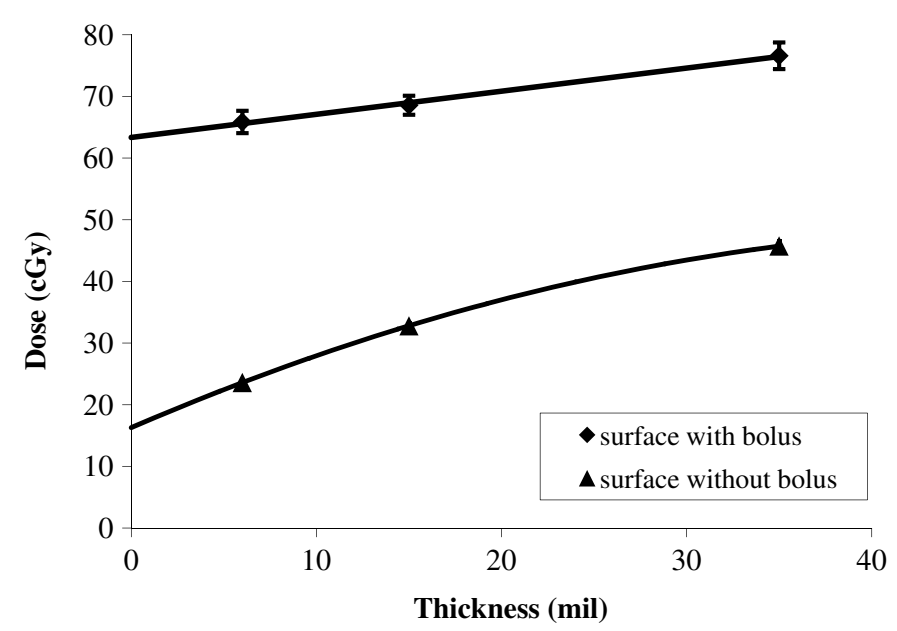

Figure 2. The dose as a function of the TLD thickness for an example set of measurements corrected with the TLD extrapolation method using quadratic (surface dose without bolus) and linear (dose below bolus) methods.

\subsection{The verification of the TLD extrapolation method}

The TLD extrapolation method was validated by measuring the percent depth dose (PDD) for a $5 \times 5 \mathrm{~cm}^{2}$ field with TLDs and the Attix chamber in the slab phantom at a $90 \mathrm{~cm}$ source-tosurface distance (SSD). Measurements were made with 100 MU. Depth doses were measured along the central axis at multiple depths at $0^{\circ}$ and $45^{\circ}$ incident angles $\left(0^{\circ}: 0,0.2,0.5,1.0,1.5\right.$, $10 \mathrm{~cm} ; 45^{\circ}: 0,0.3,0.6,1.0,1.4,10 \mathrm{~cm}$ (slant depth)). Then, the dose was normalized to the dose at a $10 \mathrm{~cm}$ depth for each incident angle. For depths of $1 \mathrm{~cm}$ and deeper, only $35 \mathrm{mil}$ thicknesses of TLDs were used. The differences between 35 mil TLDs and extrapolation results were less than $2 \%$ at the deeper depths.

\subsection{Surface dose measurements on the slab phantom}

The surface doses at the central axis without bolus and with different bolus materials were measured using the Attix chamber in the slab phantom for $10 \times 10 \mathrm{~cm}^{2}$ and $10 \times 20 \mathrm{~cm}^{2}$ fields at $0^{\circ}, 45^{\circ}$ and $70^{\circ}$ incident angles at a $90 \mathrm{~cm}$ source-to-detector distance (SDD) as shown in figure 3. The SDD was held fixed with bolus materials placed above the detector. TLDs were also used in a selected number of measurements $\left(10 \times 10 \mathrm{~cm}^{2}\right.$ field at $0^{\circ}$ and $45^{\circ}$ incident angles) to permit additional comparisons of the TLD extrapolation method to the Attix chamber measurements. The dimension of the TLD was larger than the size of perforation (where applicable), so the measured dose with TLDs was the average dose below the perforated bolus, which was consistent with the Attix chamber measurement. The dose was normalized to the dose at a $10 \mathrm{~cm}$ depth for a $10 \times 10 \mathrm{~cm}^{2}$ field at a $0^{\circ}$ incidence at a $90 \mathrm{~cm}$ SSD. The two repeated measurements using the TLD extrapolation method were taken when the TLD results differed significantly from Attix chamber results ( $>3 \%)$. Other measurement points were checked using 35 mil TLDs.

\subsection{Surface dose measurements on the Rando phantom}

Custom bolus of solid, fine mesh and large mesh Aquaplasts was made to fit the chest wall region of the anthropomorphic phantom. The phantom was CT scanned ( $5 \mathrm{~mm}$ slices) with 


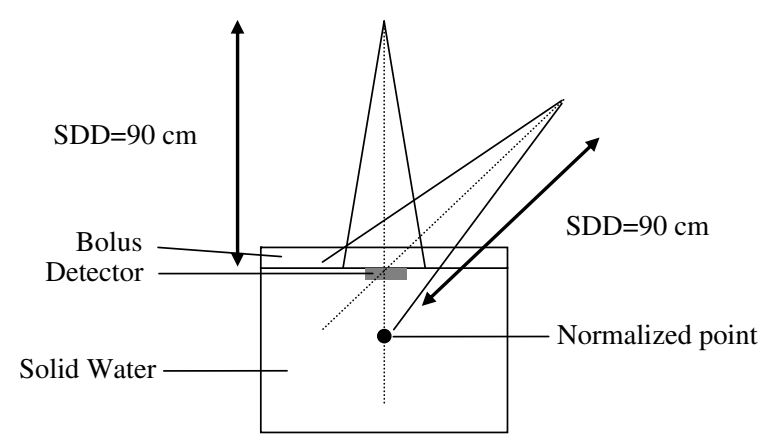

Figure 3. Experimental setup of the detector in the slab phantom for measurements at perpendicular and oblique incidences. The detector position was fixed at a $90 \mathrm{~cm}$ SDD. Bolus materials were placed above the detector. The dose normalized point was at a $10 \mathrm{~cm}$ depth for a $10 \times 10 \mathrm{~cm}^{2}$ field at a $0^{\circ}$ incidence at a $90 \mathrm{~cm} \mathrm{SSD.}$

the custom bolus pieces in place. Catheters and alignment marks were placed on the phantom. Treatment plans were created using the University of Michigan treatment planning system (UMPLAN). The edge-based calculation model (Fraass et al 1987, McShan and Fraass 1993) and convolution/superposition algorithm were used for conventional and IMRT calculations, respectively. Conventional and IMRT plans were generated using $6 \times 12 \mathrm{~cm}^{2}$ tangential fields at beam angles of $303^{\circ}$ (medial field) and $126^{\circ}$ (lateral field) to simulate a chest wall treatment for a prescribed dose of $200 \mathrm{cGy}$. For the conventional plan, a $15^{\circ}$ wedge was used on the lateral beam. For the IMRT plan, the patient surface, simulated chest wall volume and lungs were contoured and a cost function was applied using the University of Michigan optimization system (UMOpt). The chest wall volume was edited $5 \mathrm{~mm}$ from the surface (Hong et al 1999) for the optimization process to minimize the effect of known inverse planning-specific problems and inaccuracies of dose calculations in the near-surface regions. The region from the surface to $5 \mathrm{~mm}$ was excluded from the volumes used for optimization. In addition, to ensure adequate coverage of the surface, conventional flash was simulated for the IMRT beams by extending beamlet values from the tissue region to the surface region and air. Therefore, the beamlet distributions near the surface region mimicked the situation that would be required for patient treatments. The IMRT fields were sequenced for a segmental delivery (SMLC) resulting in 52 and 54 segments for medial and lateral fields, respectively. The planned dose distributions were similar between IMRT and conventional plans.

The anthropomorphic phantom was initially aligned on the treatment couch using lasers and then minor adjustments were performed using orthogonal portal images which were compared with the digital reconstructed radiographs (DRR) constructed from the treatment planning CT images to confirm the treatment position. The measured points were marked on the phantom. The surface doses were measured at three locations: the central axis of the medial field (point 1), a tangential point (point 2) and the central axis of the lateral field (point 3). At point 1, surface doses without and with bolus (Superflab and solid, fine, and large mesh Aquaplasts) were measured. At points 2 and 3, only surface doses without and with fine mesh Aquaplast were measured for additional detailed comparisons. At point 1 , the two repeated measurements using the TLD extrapolation method were taken when the variation of repeated measurements using only 35 mil TLDs was too large (>3\%). At points 2 and 3, no repeated measurements were done except at point 2 without bolus for the IMRT technique. Figure 4 shows the measured points on an axial CT image and with respect to the treatment field intensity maps for each beam. To convert TLD response to dose, three 35 mil TLDs in the 


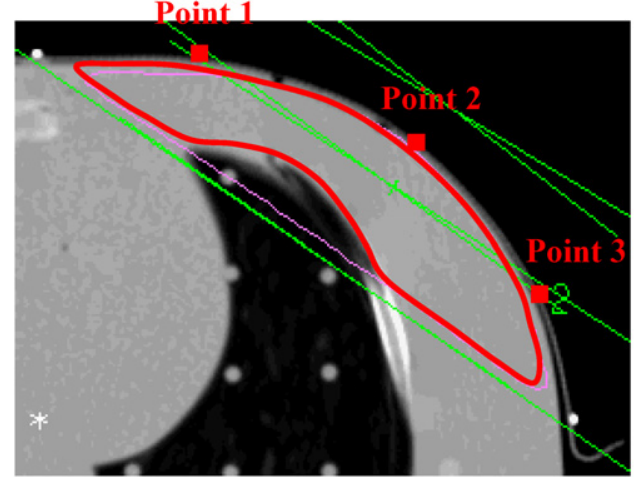

(a)
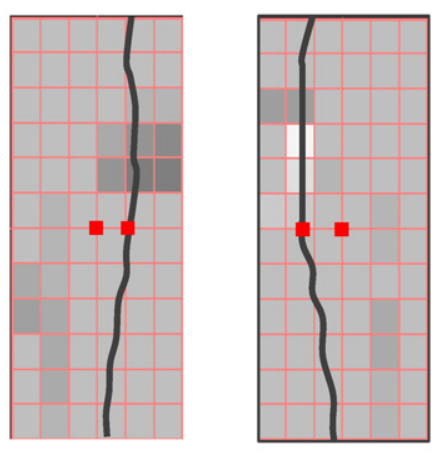

(b)

Figure 4. (a) Example axial CT image for the anthropomorphic phantom with fine mesh Aquaplast in place. (b) Fluence maps of the medial (left) and lateral (right) fields with the body surface outline shown. Whiter color means higher intensity.

slab phantom were irradiated with $80 \mathrm{cGy}$ at the calibration point to get the average dose conversion factor (TLD response/dose) for a batch of TLDs for each set of measurements to account for the day-to-day variation of the TLD reader. The TLD doses were calculated using the average dose conversion factor with each TLD's individual sensitivity factor. Finally, the measured TLD doses were divided by the prescribed fraction dose (200 cGy) to normalize the results for comparison.

\section{Results}

\subsection{The verification of the TLD extrapolation method}

Table 2 shows the measured PDDs (relative to $10 \mathrm{~cm}$ depth for an individual incident angle) using the Attix chamber and TLD extrapolation method for a $5 \times 5 \mathrm{~cm}^{2}$ field at $0^{\circ}$ and $45^{\circ}$ incidences. The dose increases rapidly at shallow depths for both incident angles and the surface dose is larger at the higher incident angle. Comparing TLD extrapolation and Attix chamber results, the local differences were found to be within $2 \%$ and $3 \%$ for $0^{\circ}$ and $45^{\circ}$ incidences, respectively (except for the surface at a $45^{\circ}$ incidence). However, the absolute difference at the surface for a $45^{\circ}$ incidence was only $3 \%$. This difference was acceptable for surface dose measurements. Therefore, the accuracy of the TLD extrapolation method was determined to be valid for measuring the near-surface dose for perpendicular and oblique incidences.

\subsection{Surface dose measurements on the slab phantom}

Table 3 shows the surface dose without bolus and with different bolus materials measured with the Attix chamber for $10 \times 10$ and $10 \times 20 \mathrm{~cm}^{2}$ fields at $0^{\circ}, 45^{\circ}$ and $70^{\circ}$ incidences. Surface dose without bolus is much smaller than that with bolus (up to $73 \%$ absolute difference for $2 \mathrm{~mm}$ fine mesh Aquaplast). Therefore, a small thickness of the bolus material will lead to a significant increase in the surface dose. For the same physical thickness, the surface doses for superflab and solid Aquaplast are within 2\% of those measured for solid water. The dose characteristics (depth and angle of incidence) of superflab and solid Aquaplast are similar to that of solid water. For the fine and large mesh bolus materials, the surface doses differed 
Table 2. PDDs measured with the Attix chamber and the TLD extrapolation method for $6 \mathrm{MV}$ beams and $5 \times 5 \mathrm{~cm}^{2}$ field at $0^{\circ}$ and $45^{\circ}$ incidences.

\begin{tabular}{cccc}
\hline Depth $(\mathrm{cm})$ & Attix $(\%)$ & TLD $(\%)$ & Local difference $(\%)$ \\
\hline \multicolumn{4}{c}{$0^{\circ}$ incident angle } \\
0.0 & 15.0 & $15.2^{\mathrm{a}}$ & 1.6 \\
0.2 & 93.5 & 92.9 & -0.6 \\
0.5 & 136.4 & 137.6 & 0.9 \\
1.0 & 160.3 & $162.0^{\mathrm{b}}$ & 1.1 \\
1.5 & 164.2 & $165.6^{\mathrm{b}}$ & 0.8 \\
10.0 & 100.0 & $100.0^{\mathrm{b}}$ & 0.0 \\
& & & \\
& & $45^{\circ}$ & incident angle \\
0.0 & 22.2 & $25.2^{\mathrm{a}}$ & 13.6 \\
0.3 & 112.0 & 112.8 & 0.7 \\
0.6 & 142.8 & 145.2 & 1.7 \\
1.0 & 158.5 & $163.0^{\mathrm{b}}$ & 2.8 \\
1.4 & 162.9 & $165.5^{\mathrm{b}}$ & 1.6 \\
10.0 & 100.0 & $100.0^{\mathrm{b}}$ & 0.0
\end{tabular}

${ }^{a}$ Quadratic extrapolation was used for the TLD extrapolation method.

b Only 35 mil TLDs were used. The differences between 35 mil TLDs and extrapolation results were estimated to be less than $2 \%$ at these depths.

Table 3. Surface doses in the slab phantom with and without bolus materials measured with the Attix chamber for $10 \times 10$ and $10 \times 20 \mathrm{~cm}^{2}$ fields at $0^{\circ}, 45^{\circ}$ and $70^{\circ}$ incidences. Data are expressed as the percentage of the dose for a $10 \times 10 \mathrm{~cm}^{2}$ field at a $10 \mathrm{~cm}$ depth and $0^{\circ}$ incidence.

\begin{tabular}{|c|c|c|c|c|c|c|c|}
\hline \multirow[b]{2}{*}{$\begin{array}{l}\text { Phantom/ } \\
\text { bolus }\end{array}$} & \multirow[b]{2}{*}{$\begin{array}{l}\text { Thickness } \\
(\mathrm{mm})\end{array}$} & \multicolumn{2}{|c|}{$0^{\circ}$ incidence } & \multicolumn{2}{|c|}{$45^{\circ}$ incidence } & \multicolumn{2}{|c|}{$70^{\circ}$ incidence } \\
\hline & & $\begin{array}{l}10 \times 10 \mathrm{~cm}^{2} \\
(\%)\end{array}$ & $\begin{array}{l}10 \times 20 \mathrm{~cm}^{2} \\
(\%)\end{array}$ & $\begin{array}{l}10 \times 10 \mathrm{~cm}^{2} \\
(\%)\end{array}$ & $\begin{array}{l}10 \times 20 \mathrm{~cm}^{2} \\
(\%)\end{array}$ & $\begin{array}{l}10 \times 10 \mathrm{~cm}^{2} \\
(\%)\end{array}$ & $\begin{array}{l}10 \times 20 \mathrm{~cm}^{2} \\
(\%)\end{array}$ \\
\hline None & 0 & 19.6 & 26.6 & 27.0 & 34.8 & 48.2 & 56.7 \\
\hline Solid water & 2 & 94.6 & 101.5 & 111.9 & 118.2 & 135.6 & 140.4 \\
\hline Solid water & 3 & 113.9 & 120.2 & 129.3 & 134.8 & 147.0 & 151.4 \\
\hline Solid water & 5 & 135.4 & 140.8 & 146.6 & 151.2 & 155.3 & 159.2 \\
\hline Solid water & 10 & 156.4 & 160.1 & 159.2 & 162.6 & 154.5 & 158.2 \\
\hline Superflab & 5 & 138.2 & 143.5 & 149.0 & 153.3 & 155.9 & 159.6 \\
\hline Superflab & 10 & 157.0 & 160.5 & 159.9 & 163.1 & 153.7 & 157.3 \\
\hline Solid Aquaplast & 2 & 92.7 & 99.2 & 109.1 & 116.5 & 134.6 & 139.1 \\
\hline Solid Aquaplast & 5 & 137.1 & 142.3 & 148.1 & 152.4 & 156.0 & 159.6 \\
\hline Fine mesh & 2 & 75.8 & 83.3 & 93.1 & 99.5 & 121.4 & 126.2 \\
\hline Large mesh & 3.2 & 97.3 & 103.9 & 114.0 & 120.0 & 136.5 & 140.8 \\
\hline
\end{tabular}

by up to $20 \%$ when compared to solid water. This difference is due to the perforations in the mesh materials which result in lower average surface doses. Water-equivalent thicknesses were estimated by comparing the depth doses with the solid water material. The estimated thicknesses were $2.1 \mathrm{~mm}$ and $1.5 \mathrm{~mm}$ for $3.2 \mathrm{~mm}$ large mesh and $2 \mathrm{~mm}$ fine mesh Aquaplast materials, respectively.

The surface dose increases with increasing incident angles. Figure 5 shows a rapid increase beyond $45^{\circ}$ incidence angle. Similar behavior was seen for all bolus materials, except for the $10 \mathrm{~mm}$ Superflab and $10 \mathrm{~mm}$ solid water slab (not shown in figure 5). The slant depth of the $10 \mathrm{~mm}$ Superflab and solid water slab at a $45^{\circ}$ incidence angle is close to the depth of $D_{\max }$, so the surface dose decreases for a $70^{\circ}$ incidence angle. Moreover, oblique incidence 


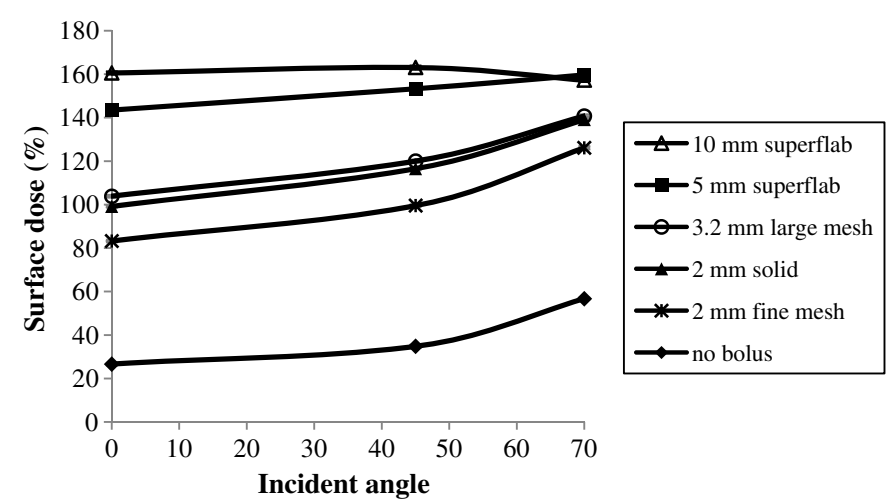

Figure 5. The behavior of surface dose with incident angle for a $10 \times 20 \mathrm{~cm}^{2}$ field.

Table 4. Surface doses in a slab phantom with and without bolus materials using the TLD extrapolation method for a $10 \times 10 \mathrm{~cm}^{2}$ field at $0^{\circ}$ and $45^{\circ}$ incidences. Data are expressed as the percentage of the dose for a $10 \times 10 \mathrm{~cm}^{2}$ field at a $10 \mathrm{~cm}$ depth and $0^{\circ}$ incidence.

\begin{tabular}{lcc}
\hline Bolus & $0^{\circ}$ incidence $(\%)$ & $45^{\circ}$ incidence $(\%)$ \\
\hline None & $20.4^{\mathrm{a}}$ & $29.9^{\mathrm{a}}$ \\
$5 \mathrm{~mm}$ superflab & 140.6 & $156.3^{\mathrm{b}}$ \\
$2 \mathrm{~mm}$ solid Aquaplast & 93.7 & $113.8^{\mathrm{b}}$ \\
$2 \mathrm{~mm}$ fine mesh & $80.4^{\mathrm{b}}$ & $96.7^{\mathrm{b}}$ \\
$3.2 \mathrm{~mm}$ large mesh & 99.9 & 116.6 \\
\hline
\end{tabular}

${ }^{a}$ Quadratic extrapolation was used for the TLD extrapolation method.

$\mathrm{b}$ The average of two repeated measurements. The average variation was $\sim 1 \%$.

has a larger influence on the surface when no bolus is present (up to $146 \%$ increase from $0^{\circ}$ to $70^{\circ}$ incidences for a $10 \times 10 \mathrm{~cm}^{2}$ field), and the influence decreases with increasing thickness of bolus.

Table 4 shows the surface dose using the TLD extrapolation method for $10 \times 10 \mathrm{~cm}^{2}$ fields at $0^{\circ}$ and $45^{\circ}$ incidences. Comparing the surface dose using the Attix chamber and TLD extrapolation, TLD doses were found to be $\sim 3 \%$ and $\sim 5 \%$ higher than those measured with the Attix chamber for $0^{\circ}$ and $45^{\circ}$ incidences, respectively. The average variation of two repeated extrapolation measurements was $\sim 1 \%$. For other measurements using the 35 mil TLDs to check reproducibility, the average variation was found to be approximately $2 \%$.

\subsection{Surface dose measurements for conventional tangential and IMRT tangential fields}

Table 5 shows the surface doses (as a percentage) using the TLD extrapolation method at three points (central axis of the medial field, tangential point and central axis of the lateral field) on the anthropomorphic phantom using the bolus materials for conventional and IMRT tangential fields. Compared to conventional tangential fields, the surface doses of IMRT fields were $3 \%$ less and $\sim 2 \%$ larger at points 1 and 2 , respectively. There was no consistent bias at point 3 . Overall, there is no significant difference between the conventional and IMRT techniques studied because the average variation was within $3 \%$ for all three points.

For the conventional tangential fields, surface doses of solid, fine mesh and large mesh Aquaplasts were $12 \%, 21 \%$ and $10 \%$ less than that of superflab, respectively. For IMRT fields, surface doses of solid, fine mesh and large mesh Aquaplasts were 14\%, 24\% and 12\% 
Table 5. Surface doses at three points in the anthropomorphic phantom with various bolus materials using the TLD extrapolation method for conventional and IMRT tangential fields. The data are expressed in the percentage of the prescribed dose, $200 \mathrm{cGy}$.

\begin{tabular}{lccl}
\hline Bolus & $\begin{array}{l}\text { Conventional } \\
(\%)\end{array}$ & $\begin{array}{l}\text { IMRT } \\
(\%)\end{array}$ & $\begin{array}{l}\text { IMRT versus } \\
\text { Conventional (\%) }\end{array}$ \\
\hline Point 1 (central axis of medial field) & & & \\
$\quad$ None & $46.4^{\mathrm{a}}$ & $45.9^{\mathrm{a}}$ & -0.5 \\
$5 \mathrm{~mm}$ superflab & 106.2 & 104.4 & -1.8 \\
$2 \mathrm{~mm}$ stretched solid Aquaplast & $93.8^{\mathrm{b}}$ & $89.9^{\mathrm{b}}$ & -3.9 \\
$2 \mathrm{~mm}$ stretched fine mesh & $84.0^{\mathrm{b}}$ & $79.8^{\mathrm{b}}$ & -4.2 \\
$3.2 \mathrm{~mm}$ stretched large mesh & $96.0^{\mathrm{b}}$ & 92.4 & -3.6 \\
Point 2 (tangential point) & & & \\
$\quad$ None & $62.1^{\mathrm{a}}$ & $63.3^{\mathrm{a}, \mathrm{c}}$ & 1.2 \\
2 mm stretched fine mesh & 105.3 & 106.6 & 1.3 \\
Point 3 (central axis of lateral field) & & & \\
$\quad$ None & $45.1^{\mathrm{a}}$ & $43.6^{\mathrm{a}}$ & -1.5 \\
$2 \mathrm{~mm}$ stretched fine mesh & 81.0 & 83.5 & 2.5 \\
\hline
\end{tabular}

${ }^{a}$ Quadratic extrapolation was used for the TLD extrapolation method.

$\mathrm{b}$ The average of two repeated measurements. The local variations were less than $3 \%$.

${ }^{\mathrm{c}}$ The average of two repeated measurements. The local variation was $9 \%$.

less than that of superflab, respectively. According to the results in section 3.2, surface doses of solid, fine mesh and large mesh Aquaplasts were 14\%, 22\% and 12\% less than that of $5 \mathrm{~mm}$ superflab for a $10 \times 10 \mathrm{~cm}^{2}$ field at a $70^{\circ}$ incidence. The dose differences measured in conventional and IMRT tangential fields were consistent with those measured in the open field (within 2\% difference). Surface doses without bolus range from $45 \%$ to $62 \%$ and $44 \%$ to $63 \%$ of the prescribed dose for conventional and IMRT tangential techniques, respectively. With bolus, the doses range from $81 \%$ to $106 \%$ and $80 \%$ to $107 \%$ of the prescribed dose for conventional and IMRT techniques, respectively.

Comparing the surface doses at different points, the surface doses at points 1 and 3 are found to be within a $5 \%$ local difference ( $4 \%$ absolute difference), but the surface doses at points 1 and 2 are between a $25 \%$ and $38 \%$ local difference (16-27\% absolute difference), depending on the use of bolus and delivery technique. The larger difference in the measurements at the tangential point (2) compared to the other points may be attributed to the larger tangential angle of incidence, along with a larger measurement uncertainty. The local variation of two repeated extrapolation measurements was $9 \%$ at the tangential point (2) without bolus for the IMRT field. This result also confirmed the large measurement uncertainty at the tangential point. At point 1 , the average variation of two repeated extrapolation measurements was $\sim 1 \%$. Local variations were less than $3 \%$.

\section{Discussion}

\subsection{The verification of the TLD extrapolation method}

The TLD extrapolation method is a useful method to estimate surface and near-surface doses in an anthropomorphic geometry. In this work, doses determined using the TLD extrapolation method were somewhat larger than those measured with an Attix chamber. A correction of TLD extrapolation results with respect to the Attix chamber results may be possible under simple conditions but may not be applicable under more complex conditions, e.g. the IMRT tangential plan. To reduce these discrepancies, a higher order fit to the extrapolation data may 
be necessary. This finding is similar to that of Kron et al (1993). Further improvements can be made to the extrapolation method by using TLD powder extrapolation (Rapley 2006). Another possible cause of the discrepancy between TLDs and the Attix chamber is the uncertainty of the over-response correction for the Attix chamber. However, the accuracy of the TLD extrapolation method is sufficient for near-surface dose estimation for perpendicular and oblique incidences, when compared with the discrepancies between treatment planning calculations and measurements in the buildup region (Fraass et al 1998).

\subsection{Surface dose measurements on the slab phantom}

Dose characteristics of Superflab and solid Aquaplast were within $2 \%$ of the solid water material. The water-equivalent thickness scaling factors of large mesh and fine mesh Aquaplasts were calculated to be 0.68 and 0.82 , respectively. The scaling factor is defined as the ratio of the water-equivalent thickness to the physical thickness (the thickness after stretching if applicable). The scaling factor is smaller for the bolus with larger holes in this study, so the average surface dose decreases with increasing size of the hole for the same physical thickness. The scaling factor may depend on the composition, density, percentage of the perforated area, and the size of perforation. For example, Mellenberg (1995) measured the surface dose for a material with bulk densities of $1.15 \mathrm{~g} \mathrm{~cm}^{-3}$ pre-stretching and $0.23 \mathrm{~g}$ $\mathrm{cm}^{-3}$ after perforation and stretching. A scaling factor of 0.3 was calculated for the work by Mellenberg (1995) and is less than that in our study. Hadley et al (2005) evaluated surface doses for head-and-neck masks with small and large perforations manufactured by Sinmed. Scaling factors for that study were calculated to be from 0.94 to 0.5 and from 0.77 to 0.37 for small-hole and large-hole masks, respectively, depending on how much the bolus was stretched. Stretching the bolus material reduces the thickness and increases the perforation size. Thus, the scaling factor decreases with increased stretching of the material. These studies demonstrate the sensitivity of the surface dose with the type, thickness, perforation size and stretching amount of bolus materials. It is recommended that a dose response estimate for the skin surface be made for various types of bolus materials prior to clinical use. With respect to the increase in the surface dose with increasing angle of incidence, the results of this study are similar to other studies (Dogan and Glasgow 2003, Lin et al 2001, Quach et al 2000, Stathakis et al 2006, Xiang et al 2007).

\subsection{Surface dose measurements for conventional tangential and IMRT tangential fields}

Previous studies have compared skin doses between conventional and IMRT techniques for head-and-neck cases. Lee et al (2002) measured the skin dose for their EF-IMRT plan with the skin as part of the target volume that was $19 \%$ and $27 \%$ larger than that of an opposed-lateral plan with and without the mask, respectively. However, when the target volume was edited $5 \mathrm{~mm}$ away from the skin, the skin dose for the EF-IMRT plan was $1 \%$ and $4 \%$ less than that of the opposed-lateral plan with and without the mask, respectively. Higgins et al (2007) found that the skin dose for an 8-field IMRT plan with the target volume restricted to $3 \mathrm{~mm}$ from the skin was $19 \%$ larger than that measured for a bilateral field technique. For chest wall tangential treatment, our studies showed that surface and near-surface doses were within a $3 \%$ difference for the conventional and IMRT techniques. The dose difference between the two delivery techniques is smaller compared with that in the study by Higgins et al (2007), but is similar to that in the study by Lee et al (2002) with the target volume $5 \mathrm{~mm}$ away from the skin for head-and-neck cases. Therefore, differences of the skin dose between delivery techniques may depend on the treatment site, angle of incidence, the number of fields and the minimum 
depth of the target volume from the skin. The amount of modulation may also be a factor with less variation in beamlet intensities over the target region for some chest wall cases compared to head-and-neck cases.

When evaluating the effect of the bolus material, the surface dose with bolus was determined to be much larger than that without bolus as expected, e.g. increases of $69 \%$ (at the tangential point) and 82\% (at the central axis points) with $2 \mathrm{~mm}$ fine mesh Aquaplast. In comparison to a study by Quach et al (2000), they found that the surface dose with $1 \mathrm{~cm}$ bolus increased by $54 \%$ (at the tangential point) and $112 \%$ (at the central axis point) compared to the dose without bolus on a chest wall phantom. Because of the different thicknesses of the bolus material used, the increase rates were different from Quach et al. However, the bolus effect was consistently larger at the central axis points than at the tangential point. Comparing the bolus effect in head-and-neck cases, Lee et al (2002) found that the skin dose increased by $\sim 18 \%$ with a thermoplastic mask. The effect of the bolus material on the surface dose is much larger for chest wall tangential radiotherapy than for head-and-neck radiotherapy. This may be due to the use of fewer beams and the different geometry for chest wall radiotherapy. Because of the sensitivity of the skin dose to the bolus placement, it is important to place bolus of suitable thickness on the chest wall to ensure sufficient dose to the tumor bed near and at the surface for chest wall radiotherapy.

For the surface dose comparison at different locations, the surface dose is greater at the tangential point for both techniques. The dose difference between the tangential and central axis points is $25-38 \%$ (16-27\% absolute) depending on the use of bolus and delivery technique. Quach et al (2000) found that the maximum surface dose was at the most oblique beam angle, e.g. tangential point. The dose at this point differs $\sim 55 \%$ without bolus when compared with the dose at the central axis point and decreases to $\sim 12 \%$ with $1 \mathrm{~cm}$ of bolus. Based on these two studies, there is in fact a distribution of dose at the surface that depends on the curvature of the treated region and the obliquity of the beams used for treatment. Regions at a large incident angle receive higher dose. Dose coverage and uniformity can be improved through the use of bolus. If improved dose homogeneity at the surface is clinically necessary, further assessments could be made using two-dimensional dosimeters and variable thicknesses of bolus.

For the conventional plan, the surface dose at point 3 (with wedge) is slightly less than that at point 1 (without wedge). The surface dose can be reduced by a wedge because medium atomic number (30-80) absorbers can reduce the secondary electron scatter in the forward direction (Khan 2003). Zhu and Palta (1998) calculated the electron contamination in 8 and $18 \mathrm{MV}$ photon beams and found that the contamination electron dose for the wedge field is less than that for an open field due to attenuation of contaminating electrons from the head by the external wedge. Moreover, the number of contaminant electrons generated in the wedge that reached the central axis is smaller than that from the flattening filter and monitor chamber. The primary component of the surface dose is electron contamination, so the surface dose in the wedge field is less than that in the open field.

\section{Conclusions}

A TLD extrapolation method was used and validated with acceptable accuracy to quantify the surface dose for different bolus types and delivery techniques. The dose characteristics of superflab and solid Aquaplast were within 2\% compared to solid water. An advantage to using Aquaplast is that it can be customized to the patient shape and ideally positioned reproducibly. By using materials with perforations, lower surface doses can be obtained. Oblique incidence 
has a larger influence on the surface when no bolus is present and this influence decreases with increasing thickness of bolus.

When evaluating the surface dose on the chest wall region of an anthropomorphic phantom, no significant difference was found between IMRT and conventional techniques. Surface doses were found to be $45-62 \%$ and $44-63 \%$ of the prescribed dose without bolus for conventional and IMRT tangential techniques, respectively. The use of bolus increased the doses to 81$106 \%$ and $80-107 \%$ of the prescribed dose for conventional and IMRT tangential fields, respectively. The effect of bolus on the surface dose was large for chest wall tangential radiotherapy, up to an $82 \%$ increase, when $2 \mathrm{~mm}$ fine mesh Aquaplast was used. Comparing the surface doses at different points, the surface dose is found to be the largest at the tangential point for both techniques (up to $38 \%$ difference). This difference decreases when bolus is used due to the increase in depth.

Technique differences were not significant when compared to the effect of incident angle and bolus thickness for the surface dose. These results can be used by clinicians to choose an appropriate bolus type and thickness for the duration of chest wall treatment. However, it should be clarified that these results provide a representative sampling because the equivalent bolus effect is dependent upon how much the bolus material is stretched. Individual clinics may need to estimate the range of surface dose as a function of the stretched bolus material to determine the doses for their own practice. Further work is needed to quantify the distribution of surface dose in two dimensions for a chest wall geometry and treatment technique, and to determine the discrepancies between calculations and measurements in the buildup region. This information can then be used to improve dose calculation models in the buildup region.

\section{References}

Biggs P J and Ling C C 1979 Electrons as the cause of the observed $d_{\max }$ shift with field size in high energy photon beams Med. Phys. 6 291-5

Dogan N and Glasgow G P 2003 Surface and build-up region dosimetry for obliquely incident intensity modulated radiotherapy $6 \mathrm{MV}$ x rays Med. Phys. 30 3091-6

Fraass B, Doppke K, Hunt M, Kutcher G, Starkschall G, Stern R and Van Dyke J 1998 AAPM task group 53: quality assurance for clinical radiotherapy treatment planning Med. Phys. 25 1773-822

Fraass B A, McShan D L, Ten Haken R K and Hutchins K M 1987 The Use of Computers in Radiation Therapy ed I A D Bruinvis et al (Amsterdam: Elsevier) pp 521-4

Gerbi B J 1993 The response characteristics of a newly designed plane-parallel ionization chamber in high-energy photon and electron beams Med. Phys. 20 1411-5

Gerbi B J and Khan F M 1990 Measurement of dose in the buildup region using fixed-separation plane-parallel ionization chambers Med. Phys. 17 17-26

Gerbi B J and Khan F M 1997 Plane-parallel ionization chamber response in the buildup region of obliquely incident photon beams Med. Phys. 24 873-8

Gerbi B J, Meigooni A S and Khan F M 1987 Dose buildup for obliquely incident photon beams Med. Phys. 14 393-9

Hadley S W, Kelly R and Lam K 2005 Effects of immobilization mask material on surface dose J. Appl. Clin. Med. Phys. 6 1-7

Higgins P D, Han E Y, Yuan J L, Hui S and Lee C K 2007 Evaluation of surface and superficial dose for head and neck treatments using conventional or intensity-modulated techniques Phys. Med. Biol. 52 1135-46

Hong L, Hunt M, Chui C, Spirou S, Forster K, Lee H, Yahalom J, Kutcher G J and McCormick B 1999 Intensitymodulated tangential beam irradiation of the intact breast Int. J. Radiat. Oncol. Biol. Phys. 44 1155-64

Khan F M 2003 The Physics of Radiation Therapy 3rd edn, ed F M Khan (Philadelphia: Lippincott Williams \& Wilkins) p 283

Kron T, Butson M, Hunt F and Denham J 1996 TLD extrapolation for skin dose determination in vivo Radiother. Oncol. 41 119-23

Kron T, Elliot A, Wong T, Showell G, Clubb B and Metcalfe P 1993 X-ray surface dose measurements using TLD extrapolation Med. Phys. 20 703-11 
Lamb A and Blake S 1998 Investigation and modeling of the surface dose from linear accelerator produced 6 and 10 MV photon beams Phys. Med. Biol. $431133-46$

Lee N, Chuang C, Quivey J M, Phillips T L, Akazawa P, Verhey L J and Xia P 2002 Skin toxicity due to intensity modulated radiotherapy for head-and-neck carcinoma Int. J. Radiat. Oncol. Biol. Phys. 53 630-7

Lin J P, Chu T C, Lin S Y and Liu M T 2001 Skin dose measurement by using ultra-thin TLDs Appl. Radiat. Isot. 55 383-91

Lopez Medina A, Teijeiro A, Garcia J, Esperon J, Terron J A, Ruiz D P and Carrion M C 2005 Characterization of electron contamination in megavoltage photon beams Med. Phys. 32 1281-92

McShan D L and Fraass B A 1993 Use of an octree-like geometry for 3-D dose calculations Med. Phys. 20 1219-27

Mellenberg D E 1995 Dose behind various immobilization and beam-modifying devices Int. J. Radiat. Oncol. Biol. Phys. 32 1193-7

Petti P L, Goodman M S, Gabriel T A and Mohan R 1983a Investigation of buildup dose from electron contamination of clinical photon beams Med. Phys. 10 18-24

Petti P L, Goodman M S, Sisterson J M, Biggs P J, Gabriel T A and Mohan R 1983b Sources of electron contamination for the clinic-35 25-MV photon beam Med. Phys. 10 856-61

Quach K Y, Morales J, Butson M J, Rosenfeld A B and Metcalfe P E 2000 Measurement of radiotherapy X-ray skin dose on a chest wall phantom Med. Phys. 27 1676-80

Rapley P 2006 Surface dose measurement using TLD powder extrapolation Med. Dosim. 31 209-15

Rawlinson J A, Arlen D and Newcombe D 1992 Design of parallel plate ion chambers for buildup measurements in megavoltage photon beams Med. Phys. 19 641-8

Stathakis S, Li J S, Paskalev K, Yang J, Wang L and Ma C M 2006 Ultra-thin TLDs for skin dose determination in high energy photon beams Phys. Med. Biol. 51 3549-67

Thomas S J and Hoole A C 2004 The effect of optimization on surface dose in intensity modulated radiotherapy (IMRT) Phys. Med. Biol. 49 4919-28

Xiang H F, Song J S, Chin D W, Cormack R A, Tishler R B, Makrigiorgos G M, Court L E and Chin L M 2007 Build-up and surface dose measurements on phantoms using micro-MOSFET in 6 and $10 \mathrm{MV}$ x-ray beams and comparisons with Monte Carlo calculations Med. Phys. 34 1266-73

Yang J, Li J S, Qin L, Xiong W and Ma C M 2004 Modelling of electron contamination in clinical photon beams for Monte Carlo dose calculation Phys. Med. Biol. 49 2657-73

Yokoyama S, Roberson P L, Litzenberg D W, Moran J M and Fraass B A 2004 Surface buildup dose dependence on photon field delivery technique for IMRT J. Appl. Clin. Med. Phys. 5 71-81

Yu C and Luxton G 1999 TLD dose measurement: a simplified accurate technique for the dose range from $0.5 \mathrm{cGy}$ to 1000 cGy Med. Phys. 26 1010-6

Zhu T C and Palta J R 1998 Electron contamination in 8 and 18 MV photon beams Med. Phys. 25 12-9 